\title{
A proposal for curriculum content in transfusion medicine and blood banking education in pathology residency programs
}

\author{
Mark K. Fung, Kendall P. Crookston, Gay Wehrli, Ronald Domen, Ileana Lopez-Plaza, \\ Robertson Davenport, Jerome Gottschall, and Steven Spitalnik for the AABB Taskforce on \\ Transfusion Medicine Resident Curriculum
}

$\mathrm{T}$ he fields of blood banking (BB) and transfusion medicine (TM), like other areas of medicine, have evolved into specialties with a diverse array of areas of knowledge. These areas include advances in the detection of pathogens in donated blood, advances in methods to collect and manipulate progenitor cells, advances in blood conservation techniques, and advances in treatments for both the acutely and the

ABBREVIATIONS: ACGME $=$ Accreditation Council for Graduate Medical Education; $\mathrm{BB}=$ blood banking; $\mathrm{CAP}=$ College of American Pathologists; FACT $=$ Foundation for the Accreditation of Cellular Therapy; IC = interpersonal and communication skills; JCAHO = Joint Commission on Accreditation of Healthcare Organizations; $\mathrm{MK}=$ medical knowledge; $\mathrm{PC}=$ patient care; $\mathrm{PL}=$ practice-based learning and improvement; $\mathrm{PR}=$ professionalism; $\mathrm{SP}=$ systems-based practice; $\mathrm{TM}=$ transfusion medicine.

From the Department of Pathology and Laboratory Medicine, Fletcher Allen Health Care, Burlington, Vermont; the Department of Pathology, University of Vermont, Burlington, Vermont; the Department of Pathology, University of New Mexico, Albuquerque, New Mexico; TriCore Reference Laboratories, Albuquerque, New Mexico; United Blood Services of New Mexico, Albuquerque, New Mexico; Blood Bank and Transfusion Services, Tufts-New England Medical Center, Boston, Massachusetts; the Department of Pathology, Penn State Milton S. Hershey Medical Center, Hershey, Pennsylvania; the Department of Pathology, University of Michigan Hospitals, Ann Arbor, Michigan; the Blood Center of Wisconsin, Milwaukee, Wisconsin; and the Department of Pathology, Columbia University College of Physicians \& Surgeons, New York, New York.

Address reprint requests to: Mark K. Fung, MD, PhD, Department of Pathology, Fletcher Allen Health Care, 111 Colchester Avenue, Burlington, VT 05401; e-mail: Mark.Fung@ vtmednet.org.

Received for publication February 1, 2007; revision received March 29, 2007, and accepted March 29, 2007.

doi: 10.1111/j.1537-2995.2007.01417.x

TRANSFUSION 2007;47:1930-1936. chronically ill patient, among many others. As a consequence, the knowledge base that a resident trainee in pathology is expected to master has expanded well beyond the mere understanding of compatibility testing and inventory management.

In the past several years, guidance regarding pathology residency training has been published for clinical pathology and anatomic pathology, as well as in some subspecialty areas. ${ }^{1-8}$ Although intended for medical students, an NIH-funded transfusion medicine curriculum initiative also provided a comprehensive overview of areas of knowledge relevant to pathology resident training. ${ }^{9,10} \mathrm{~A}$ survey of TM fellowships was also performed with implications for residency training, as have recent commentaries regarding the role of physicians in blood centers and hospital transfusion services. ${ }^{11-13}$ There is no document that specifically addresses pathology residency training in $\mathrm{BB}$ and/or TM, however, and the current requirements of the Accreditation Council for Graduate Medical Education (ACGME) on general competencies in residency training. ${ }^{14}$ Recently, the Academy of Clinical Laboratory Physicians and Scientists (ACLPS) and the members of the Children's Health Initiative through Laboratory Diagnostics (CHILDx) separately produced comprehensive clinical pathology residency training documents of which $\mathrm{BB}$ and/or TM was a subsection. ${ }^{3,8}$ As a follow-up to the activities of ACLPS and the new requirements of the ACGME regarding general competencies, a task force was created by the AABB to address the needs for a residency curriculum on this subject. The rotation objectives outlined below for BB and TM were adapted from the subsection on $\mathrm{BB}$ and/or TM from the ACLPS document and further expanded. The task force further elaborated on these objectives by including additional guidance on the different needs of the general anatomic pathology- and clinical pathology-trained pathologist, who has either minimal or infrequent supervision of a transfusion service, compared with the specialist whose responsibilities might include directing a blood collection center, a cell therapeutics laboratory, or a high-complexity transfusion service. The task force further designated what each specific rotation 
objective accomplished with respect to the ACGME general competencies.

In addition to the provision and further elaboration of a common set of rotation objectives to help residency programs ensure adequate depth and breadth of training in $\mathrm{BB}$ and/or TM, three particular areas were also considered by the task force: skill levels, duration of rotation, and need for time at other facilities when local training resources are limited.

\section{SKILL LEVELS}

The skill levels of the rotation objectives are roughly divided into three categories:

- Skill Level 1: Expectation of all clinical pathology residents after their initial months of training.

- Skill Level 2: Expectation of all clinical pathology residents by completion of residency training.

- Skill Level 3: Expectation for all BB and TM fellows and all other trainees who assume comparable job responsibilities afterward (e.g., hematology-oncology fellows or clinical pathology-only trainees desiring to direct a high complexity transfusion service and/or blood bank at an academic medical center).

The distribution of objectives between the different skill levels is intended as a rough guideline for the progression of a resident's acquisition of skills, knowledge, and experience in TM and/or BB. It is the task force's belief, however, that the sum of objectives in Skill Levels 1 and 2 are reasonable expectations of a resident by completion of residency training. Individual training programs vary in the length and intensity of BB and/or TM rotations and the volume and frequency of clinical material. Therefore, there will be some differences in the sequence in which some of these objectives are expected to be accomplished within each program. In addition, the resident's progression through the different areas and skill levels will vary according to individual abilities and interest. Finally, where some objectives overlap at the different skill levels, the expectation is that the resident will exercise greater independence with skills already previously acquired.

\section{DURATION OF ROTATION AND NEED FOR TIME SPENT AT OTHER FACILITIES}

The ACGME has no requirements for the minimum number of months required for any particular area of pathology training, although it does have requirements for the number of cases reviewed in other areas of pathology training. ${ }^{15}$ Furthermore, some residency programs include some aspects of hemostasis and/or coagulation testing or histocompatibility testing as part of their BB and/or TM rotation, whereas for other residency programs these are separate rotations. Therefore, an accounting of the number of months that a resident should devote to $\mathrm{BB}$ and/or TM is difficult in actual practice. The minimum requirements have ranged from as few as 2 months to as many as 5 months. The length of the overall exposure to $\mathrm{BB}$ and/or TM should vary according to the goals of the particular trainee and also based on the intensity of the local training environment. Highly complex cases might arise frequently in certain institutions, whereas other institutions may rely more heavily on case studies and formal didactic approaches to achieve the same goals. Some aspects of BB and TM training, however, can only be obtained from caring for patients or donors with special needs. In those instances where certain rotation objectives cannot be achieved with local resources, some thought should be given to making arrangements with another facility to at least provide some brief hands-on experience, particularly at Skill Level III (e.g., regional blood center, tertiary medical center, testing laboratories, apheresis service, hematopoietic stem cell [HSC] transplant unit).

Finally, two areas outside of TM should be mentioned because they are often incorporated into $\mathrm{TM}$ and/or $\mathrm{BB}$ rotations in part or in whole: histocompatibility testing and hemostasis and/or coagulation. Some resident rotation objectives in hemostasis and/or coagulation are included in this document. This is an acknowledgment that the areas of hemostasis and/or coagulation cross the boundaries of hematology, hematopathology, and TM. Depending on the nature of the expertise in a particular institution, the physician trained in TM may render services in the diagnosis and management of disorders of hemostasis and coagulation. Likewise, histocompatibility testing also interfaces with and has common origins with TM, and some histocompatibility testing objectives are included as they relate directly to TM. More detailed objectives and goals for resident training in histocompatibility testing and in hemostasis and/or coagulation can be found elsewhere. ${ }^{3}$ The task force hopes that this document will be useful for residents, fellows, and residency directors and those actively involved in the teaching of TM and/or BB.

\section{RECOMMENDED TRANSFUSION MEDICINE AND/OR BLOOD BANKING ROTATION OBJECTIVES}

ACGME general competencies fulfilled by these objectives are labeled as: professionalism [PR], patient care [PC], medical knowledge $[\mathrm{MK}]$, practice-based learning and improvement $[\mathrm{PL}]$, interpersonal and communication skills [IC], and systems-based practice [SP].

\section{Transfusion Service}

Skill Level I

- Demonstrate knowledge of the principles of patient and/or unit identification and pretransfusion testing, 
including $\mathrm{ABO}$ and/or Rh testing, red blood cell (RBC) antibody screen, and antibody identification [PC, $\mathrm{MK}]$.

- Choose appropriate crossmatching methods for various patients (e.g., electronic, immediate-spin, antiglobulin) [PC, MK].

- $\quad$ Recognize and appropriately refer serologic evaluations that are beyond the scope of a hospital-based transfusion service and/or blood bank [PC, SP].

- Describe the expected response to transfusion therapy in adult and pediatric patients [MK, $\mathrm{PC}]$.

- $\quad$ Triage and screen requests for blood components appropriately during inventory shortages [PC, SP] .

- $\quad$ Demonstrate the ability to perform blood utilization reviews [PC, SP].

- Demonstrate the ability to clinically evaluate a reported transfusion reaction and order and interpret appropriate initial laboratory testing [PC, MK, PL].

- $\quad$ Recognize the symptoms and signs of hemolytic and nonhemolytic transfusion reactions and demonstrate knowledge of the pathophysiology, treatment, and prevention of these complications [MK, PC].

- Identify the major infectious complications of blood transfusions and the current risk of these infections and explain how these infections can be prevented [MK].

- Identify the major noninfectious complications of blood transfusions, including TRALI, the risk of these complications, and strategies to prevent them [MK].

- Demonstrate knowledge of the indications for cytomegalovirus-reduced-risk blood, leukoreduction, irradiation, and washing of blood components [MK].

- $\quad$ Choose appropriate blood components and derivatives based on a thorough knowledge of the indications for transfusion [PL, PC, MK].

- Demonstrate knowledge of the pathophysiology, prevention, and treatment of hemolytic disease of the fetus and newborn. Recognize those antibodies in pregnant patients that are clinically significant and make appropriate recommendations for blood products [MK, PL, PC].

- $\quad$ Demonstrate knowledge of the potential side effects of neonatal whole-blood exchanges and massive transfusions in neonates associated with extracorporeal circuits such as used in extracorporeal membrane oxygenation or in cardiac surgery [MK, PC].

- Demonstrate knowledge of the pathophysiology and treatment of neonatal alloimmune thrombocytopenia $[\mathrm{MK}, \mathrm{PC}]$.

- Demonstrate proficiency in the evaluation and appropriate transfusion therapy of adult and pediatric thrombocytopenic patients secondary to both immune and nonimmune etiologies [PC, PL].
- Apply the principles of a massive transfusion protocol [PL, IC, SP, MK].

- $\quad$ Demonstrate a working knowledge of the principles of hemostasis and coagulation and proficiency in the initial treatment of patients with bleeding disorders [MK, PC].

- Demonstrate knowledge of the transfusion requirements of special patient populations (e.g., hematology-oncology, pediatrics, geriatrics, transplantation, burn, and/or trauma) [MK, PC].

\section{Skill Level II}

- Demonstrate knowledge of options for preventing volume overload in pediatric transfusion therapy [MK, PC, SP].

- Identify clinically significant RBC-specific antibodies from an antibody panel, determine how difficult it will be to obtain blood for this patient, and effectively communicate these results to clinicians [PC, IC].

- Demonstrate ability to distinguish clinically significant from clinically insignificant RBC-specific antibodies [MK].

- Demonstrate proficiency in evaluating and recommending treatment plans for complex transfusion reactions $[\mathrm{MK}, \mathrm{IC}, \mathrm{SP}, \mathrm{PC}]$.

- Demonstrate familiarity with the requirements of all applicable regulatory and accrediting agencies (e.g., JCAHO, CAP, AABB, FDA, FACT) [SP].

- Demonstrate competence in managing blood inventory and ability to communicate effectively the hospital's needs to the blood supplier [IC, SP].

- Demonstrate knowledge of various methods of blood conservation, including pre- and perioperative autologous blood collection, and approaches to "bloodless" surgery [MK, PL, SP].

- Demonstrate proficiency in evaluating patients refractory to platelet (PLT) transfusions, including the principles of histocompatibility testing and the roles of HLA-matched PLTs versus PLT crossmatching, and apply this knowledge in selecting appropriate PLT products when indicated [PC, MK].

- Demonstrate proficiency in evaluating patients with immune-mediated and non-immune-mediated hemolytic anemia and in the appropriate testing and transfusion management of these patients [PC, MK].

- Demonstrate ability to communicate laboratory testing results, transfusion recommendations, and blood supply issues to clinicians, both verbally and in written form [IC, SP, PC].

- $\quad$ Demonstrate ability to write an appropriate consult note for a patient who has an alloantibody, explaining the clinical significance of the finding to the treating physicians and the additional logistical requirements for obtaining compatible blood [IC, SP, PC]. 
- Demonstrate knowledge of landmark published studies in TM [MK, PL].

- Demonstrate proficiency in evaluating and presenting findings to professional colleagues from 1) recent peer-reviewed journal articles related to TM and 2) research projects in which the resident may participate [PL, IC].

- Demonstrate knowledge of how to host an inspection by a regulatory or accrediting agency [SP, IC, PR].

- Demonstrate proficiency at preparing educational presentations on TM topics and the ability to adapt presentations to audiences of differing experience levels (e.g., pathologists, nonpathology physicians, technologists, nurses, and blood center workers] [IC, $\mathrm{MK}]$.

- Differentiate between plasma-derived and recombinant factor products and demonstrate knowledge of on-label and off-label indications for these products [PC, MK, PL, SP].

\section{Skill Level III}

- Demonstrate ability to interpret difficult antibody panels including those containing multiple alloantibodies, autoantibodies, and antibodies to highfrequency antigens $[\mathrm{MK}, \mathrm{PC}]$.

- Demonstrate knowledge of specialized test methods in immunohematology including elution, absorption, and use of enzymes [MK].

- Demonstrate familiarity with the appropriate use of highly specialized blood products (e.g., granulocytes, donor lymphocyte infusions, HLA-matched PLTs, coagulation factor concentrates) [MK, PC].

- Demonstrate ability to select appropriate factor replacement product(s) including creating an appropriate dose-time schedule for a patient through collaborative discussion with the patient's primary team physician [PC, MK, IC].

- Compare and contrast the various methods of performing blood utilization review, including prospective versus retrospective review, and the role of peer review as required by JCAHO and AABB standards [SP, PL, IC].

- Demonstrate ability to perform lookback investigations [SP, IC].

- Demonstrate ability to write an error or deviation report and create a corrective and preventive action plan [IC, PL, SP].

- Demonstrate an understanding of the appropriateness of transfusion of serologically incompatible blood in selected clinical circumstances [PC, MK].

- Demonstrate familiarity with the scientific, legal, and ethical issues surrounding allocation of resources, cost containment, and the role of gatekeeper [PL, IC, PR, SP].
- Demonstrate familiarity with ethical issues in BB and TM (e.g., confidentiality as it relates to the blood donor or patient, HIV testing and reporting, a patient's right to refuse blood transfusion, the use of hematopoietic growth factors in normal donors, and informed consent) [PR, PC].

- Demonstrate knowledge of the basic principles of blood conservation and bloodless medicine and surgery programs, including specific methods of preserving RBC mass, supporting hemostasis, and adjunctive therapies that may be used when the oxygen supplied by the patient's hemoglobin is insufficient [MK, SP, PL].

- Demonstrate familiarity with the relevant regional plan for major disasters and explain the roles of the local blood supplier and the transfusion service or blood bank in this plan [SP, IC].

- Participate in an accreditation inspection of a laboratory related to $\mathrm{TM}$ and/or $\mathrm{BB}$ (this may be either an official inspection or an unofficial and/or interim inspection) [SP, PR, IC].

- Demonstrate familiarity with the requirements for proper storage, preparation, and traceability of tissue-derived grafts received and dispensed by the transfusion service or blood bank [MK, SP, PC].

\section{Blood collection, blood center, and cell processing responsibilities}

Skill Level I

- Demonstrate knowledge of current eligibility criteria for blood donors [MK].

- $\quad$ Compare and contrast the eligibility requirements for allogeneic and autologous blood donations [MK].

- $\quad$ Observe and subsequently demonstrate competency in performing a donor interview and exam, including obtaining consent to donate (e.g., risks, benefits, alternatives, and answer questions) [PC, IC, PR].

- Demonstrate knowledge of the indications for therapeutic phlebotomy [MK].

- Demonstrate proficiency in evaluating and treating adverse reactions associated with blood donation and/or phlebotomy (both whole-blood and apheresis donations) [PC, PR, MK].

- $\quad$ Outline the assay principles (e.g., nucleic acid testing, enzyme-linked immunorbent assay) of required donor blood tests and the associated confirmatory testing and describe examples of donor reentry algorithms [MK, SP].

- Demonstrate professionalism in interactions with prospective donors [PR, IC].

- Demonstrate familiarity with the steps in blood component and blood derivative preparation [MK, $\mathrm{SP}]$. 
- Describe the factors that influence the motivation of blood donors [MK, SP].

- Demonstrate knowledge of the advantages and disadvantages of directed blood donation and limited donor exposure programs [PC, MK, SP].

- Demonstrate knowledge of the techniques of safe, sterile venipuncture and the associated methods to reduce bacterial contamination of products [PC, MK].

- Demonstrate familiarity with the types and treatment of donor adverse events [PC, MK].

\section{Skill Level II}

- $\quad$ Outline the necessary steps in donor notification and counseling associated with positive infectious disease testing results and the donor lookback process [PC, SP, IC].

- Demonstrate understanding of, and the ability to interpret, the major regulations and guidelines applicable to collection, processing, storage, and release of blood products and cellular therapy products [SP, $\mathrm{MK}]$.

- Demonstrate awareness of current concerns about emerging infections in the blood supply and describe ways that blood collection centers deal with these concerns [MK, PL].

- Demonstrate familiarity with the operational logistics required to determine appropriate blood inventory for a geographic region and the process of meeting daily, weekly, and monthly collection goals [SP, IC].

- Demonstrate knowledge of the principles of HSC transplantation, including collection, processing, and storage of these products, and the indications for use (e.g., marrow, peripheral blood, and placental and/or umbilical cord blood) [MK, SP, PC].

- $\quad$ Demonstrate proficiency in writing physician orders for peripheral blood HSC collections and obtaining consent for the procedure and for blood product transfusion, if needed after the collection [PC, MK, IC, PR].

- $\quad$ Demonstrate proficiency in evaluating and treating adverse reactions associated with peripheral blood progenitor cell (PBPC) collection [PC, MK].

\section{Skill Level III}

- Demonstrate ability to review quality control data from the collecting, processing, and storage of individual HSC products and actions needed when product or processing irregularities are identified [MK, SP, PC].

- Demonstrate familiarity with emerging areas of cellular therapy, including hematopoietic graft engineering and cellular immunotherapeutics [MK, PL, PC].
- Demonstrate understanding of the elements of current good manufacturing practices and current good tissue practices as they apply to the collection, processing, ex vivo manipulation, and storage of all cellular therapy products (e.g., pancreatic islet cells, negative and/or positive selection and/or purging of HSCs, gene manipulation, donor lymphocyte infusions, dendritic cell vaccines, ex vivo expansion of progenitor cells) [MK, SP, PC].

- Demonstrate familiarity with requirements and procedures for source plasma donors [MK, SP, PC].

- Demonstrate familiarity with standards and regulations that apply to blood collection centers, clinical tissue banks, and PBPC processing laboratories (FDA, AABB, FACT, CAP) [MK, SP, PC].

- Demonstrate familiarity with quality systems as they apply to blood collection centers and PBPC processing laboratories (i.e., AABB Quality System Essentials, ISO 9000, lean manufacturing, Six Sigma) [SP, PL]

- $\quad$ Participate in quality review of the manufacturing or processing of products [PL].

\section{Therapeutic apheresis}

Skill Level I

- Summarize the principles of apheresis technology, including centrifugation, filtration, and immunoadsorption $[\mathrm{MK}]$.

- Demonstrate knowledge of the major indications for therapeutic apheresis including the category of evidence for each of these indications as outlined by AABB and/or American Society for Apheresis [MK, PC].

- Demonstrate knowledge of the appropriate replacement fluids to be used in an apheresis procedure [MK, PC].

- Demonstrate knowledge of vascular access requirements and options for therapeutic apheresis [MK, PC].

- $\quad$ Demonstrate proficiency in evaluating and preparing patients for therapeutic apheresis, including obtaining consent for the procedure and for transfusion of blood products during the procedure [IC, PC, PR].

- Communicate effectively with attending clinicians and house staff regarding emergent or scheduled therapeutic apheresis procedures through conversations and writing of consult notes [IC, SP, PR, $\mathrm{MK}]$.

\section{Skill Level II}

- Discuss the major indications for, and limitations of, therapeutic apheresis in children [PC, MK]. 
- Demonstrate ability to triage requests for therapeutic apheresis [MK, PC, IC, PR].

- $\quad$ Demonstrate proficiency in evaluating and treating adverse reactions associated with therapeutic apheresis [PC, MK, IC].

- Write appropriate physician orders for therapeutic apheresis procedures [PC, MK, IC].

\section{Skill Level III}

- Demonstrate proficiency in treating patients with specialized apheresis methods (e.g., photopheresis, immunoadsorption columns) [PC, MK].

- Demonstrate proficiency in evaluating, assessing, and treating a wide variety of patients who require therapeutic apheresis for various disorders [MK, PC, IC, PR].

- Demonstrate ability to evaluate literature for therapeutic apheresis for which the data suggesting efficacy for a particular disease entity is limited and communicate effectively with primary care physicians to develop a plan of care [MK, PC, IC, SP, PL].

\section{TRANSFUSION MEDICINE REFERENCE MATERIALS}

\section{Handbooks or introductory texts}

Harmening DM. Modern blood banking and transfusion practices. 5th ed. Philadelphia; F.A. Davis Co.; 2005.

Gottschall J, editor. Blood transfusion therapy: a physician's handbook. 8th ed. Bethesda: American Association of Blood Banks; 2005.

McLeod B, editor. Therapeutic apheresis: a physician's handbook. 1st ed. Bethesda: American Association of Blood Banks; 2005.

Roseff S, editor. Pediatric transfusion: a physician's handbook. 1st ed. Bethesda: American Association of Blood Banks; 2003.

Snyder E, Haley NR. Cellular therapy: a physician's handbook. 1st ed. Bethesda: American Association of Blood Banks; 2004.

McCullough J. Transfusion medicine. 2nd ed. New York: Churchill Livingstone; 2004.

Petrides M, Stack G. Practical guide to transfusion medicine. Bethesda: American Association of Blood Banks; 2001

Murphy M, Pamphilon D, editors. Practical transfusion medicine. 2nd ed. Boston: Blackwell Publishing; 2005.

\section{Standards, comprehensive, or special topic texts}

AABB Technical Manual. 15th ed. Bethesda: American Association of Blood Banks; 2005.
Mintz PD. Transfusion therapy: clinical principles and practice. 2nd ed. Bethesda: American Association of Blood Banks; 2005.

Standards for blood banks and transfusion services. 24th ed. Bethesda: American Association of Blood Banks; 2006.

Standards for cellular therapy product services. 2nd ed. Bethesda: American Association of Blood Banks; 2007.

McLeod BC, Price TH, Weinstein RA. Apheresis: principles and practice. 2nd ed. Bethesda: American Association of Blood Banks; 2003.

Hillyer CD, Silberstein LE, Ness PM, Anderson KC, Roback JD. Blood banking and transfusion medicine: basic principles and practice. 2nd ed. New York: Churchill Livingstone; 2007.

Klein HG, Anstee, DJ. Mollison's blood transfusion in clinical medicine. 11th ed. Boston: Blackwell; 2005.

Simon TL, Dzik WH, Snyder EL, Rossi EC, Stowell CP, Strauss RG. Rossi's principles of transfusion medicine. 3rd ed. Philadelphia: Lippincott Williams \& Wilkins; 2002.

Petz LD, Garratty G. Immune hemolytic anemias. 2nd ed. New York: Churchill Livingstone; 2004

Reid ME, Lomas-Francis C. The blood group antigen facts book. 2nd ed. New York: Elsevier; 2004

Blackall DP, Helekar PS, Triulzi DJ. Transfusion medicine: self-assessment and review. Bethesda: American Association of Blood Banks; 2002

Herman J, Manno C. Pediatric transfusion therapy. Bethesda: American Association of Blood Banks; 2002

Hillyer CD, Strauss R, Luban N. Handbook of pediatric transfusion medicine. San Diego: Elsevier Academic Press; 2004

Popovsky M, editor. Transfusion reactions. 3rd ed. Bethesda: American Association of Blood Banks; 2007.

Spiess BD, Spence, RK, Shander A. Perioperative transfusion medicine. 2nd ed. Philadelphia: Lippincott, Williams, \& Wilkins; 2005.

Issitt PD, Anstee DJ. Applied blood group serology. 4th ed. Durham (NC): Montgomery Scientific; 1998

\section{Web sites}

http://www.aabb.org/content-Accrediting organization for transfusion services, blood centers, and cell processing laboratories. Includes links to several useful resources.

http://www.fda.gov/cber/blood.htm-FDA/CBER Web page with links to other documents on regulatory issues pertaining to the production and use of blood products.

http://www.osha.gov/SLTC/

bloodbornepathogens/-OSHA Web page with general information on blood-borne pathogens, needle-stick prevention, and blood exposure in the workplace. 
http://www.apheresis.org/-American Society for Apheresis Web site.

http://www.factwebsite.org/-Foundation for the Accreditation of Cellular Therapy Web site.

http://www.cbbsweb.org/-California Blood Bank Society Web site.

http://www.sabm.org/-Society for the Advancement of Blood Management Web site.

http://www.nataonline.com-Network for Advancement of Transfusion Alternatives Web site.

\section{ACKNOWLEDGMENT}

We thank Paul Mintz, MD, who was responsible for the creation of this task force.

\section{REFERENCES}

1. Graylyn Conference Report. Recommendations for reform of clinical pathology residency training. Conjoint Task Force of Clinical Pathology Residency Training Writing Committee. Am J Clin Pathol 1995;103:127-9.

2. Curriculum content and evaluation of resident competency in anatomic pathology: a proposal. Hum Pathol 2003;34:1083-90.

3. Smith BR, Wells A, Alexander CB, Bovill E, Campbell S, Dasgupta A, Fung M, Haller B, Howe JG, Parvin C, Peerschke E, Rinder H, Spitalnik S, Weiss R, Wener M; Academy of Clinical Laboratory Physicians and Scientists. Curriculum content and evaluation of resident competency in clinical pathology (laboratory medicine): a proposal. Clin Chem 2006;52:917-49.

4. Goals and objectives for molecular pathology education in residency programs. The Association for Molecular Pathology Training and Education Committee. J Mol Diagn 1999; 1:5-15.

5. Henricks WH, Boyer PJ, Harrison JH, Tuthill JM, Healy JC. Informatics training in pathology residency programs: proposed learning objectives and skill sets for the new millennium. Arch Pathol Lab Med 2003;127:1009-18.
6. Horowitz RE, Naritoku W, Wagar EA. Management training for pathology residents: a regional approach. Arch Pathol Lab Med 2004;128:59-63.

7. Beastall G, Kenny D, Laitinen P, ten Kate J. A guide to defining the competence required of a consultant in clinical chemistry and laboratory medicine. Clin Chem Lab Med 2005;43:654-9.

8. Pysher TJ, Bach PR, Geaghan SM, Hamilton MS, Laposata M, Lockitch G, Brugnara C, Coffin CM, Pasquali M, Rinaldo P, Roberts WL, Rutledge JC, Ashwood ER, Blaylock RC, Campos JM, Goldsmith B, Jones PM, Lim M, Meikle AW, Perkins SL, Perry DA, Petti CA, Rogers BB, Steele PE, Weiss RL, Woods G. Teaching pediatric laboratory medicine to pathology residents. Arch Pathol Lab Med 2006;130: 1031-8.

9. Simon TL. Comprehensive curricular goals for teaching transfusion medicine. Curriculum Committee of the Transfusion Medicine Academic Award Group. Transfusion 1989; 29:438-46.

10. Cable RG, Thal SE, Fink A, Calhoun L, Petz LD. A comprehensive transfusion medicine curriculum for medical students. Transfusion Medicine Academic Award Group. Transfusion 1995;35:465-9.

11. Domen RE, Rybicki LA, Popovsky MA, Milam JD. Fellowship training programs in blood banking and transfusion medicine: results of a national survey. Am J Clin Pathol 1996;106:584-7.

12. McCullough J. The role of physicians in blood centers. Transfusion 2006;46:854-61.

13. Szczepiorkowski ZM, AuBuchon JP. The role of physicians in hospital transfusion services. Transfusion 2006;46: 862-7.

14. Outcome project (monograph on the Internet). Chicago (IL): ACGME; c2007 (accessed 2007 Feb 1). Available from: http://www.acgme.org/outcome/comp/compFull.asp

15. Accreditation Council for Graduate Medical Education (homepage on the Internet). Chiacgo (IL): The Council; c2000-2007 (2007 Feb 1). Pathology Program Requirements; (about 1 screen). Available from: http:// www.acgme.org/acWebsite/RRC_300/300_prIndex.asp 